\title{
Perioperative medicine: why do we care?
}

\author{
Homer Yang, MD
}

Received: 16 December 2014/ Accepted: 14 January 2015/Published online: 23 January 2015

(C) Canadian Anesthesiologists' Society 2015

In the article, "Perioperative fellowship curricula in anesthesiology: a systematic review", which appears in this issue of the Journal, the authors note that there is "a paucity of published literature on what perioperative care entails as well as what perioperative fellowships should include in their curricula". 1 This assessment of the current state of perioperative training and research is timely if we are to be prescient about the future directions of research and training in our speciality.

What is perioperative medicine and why do we care? There are three reasons why we should look closely at this emerging medical discipline: economics, the continuing trend of a decrease in patient mortality, and changing medical priorities.

\section{Economics}

In 1996, Longnecker wrote in Planning the Future of Anesthesiology that "Anesthesiologists will likely work less in the future", and predicted that "market forces [will] dominate decision-making regarding anesthesia practice...."2 Examining our practice solely on economic market forces, however, gives a misleading perspective. Though we need to be aware of economics, we are physicians first. Implicit in the privileges we receive from society is our duty to address societal medical needs.

\section{H. Yang, MD (ه)}

Department of Anesthesiology, The Ottawa Hospital, University of Ottawa, The Ottawa Hospital Civic Campus, Room B303, 1053 Carling Avenue, Mail Stop 249, Ottawa, ON K1Y 4E9, Canada

e-mail: hyang@toh.on.ca
In Canada, anesthesia is predominantly a hospital-based practice. Indeed, in a recent Canadian Institute for Health Information (CIHI) report on overall healthcare expenditures, the top three expense categories were hospitals, drugs, and physicians. ${ }^{3}$ In 1975, government funding to hospitals was almost $50 \%$ of healthcare expenditures, and this percentage has decreased steadily since that date. ${ }^{3}$ In 2011, hospital expenditure was $\$ 59$ billion or $29.5 \%$ of overall healthcare expenditures, with $90 \%$ funded by the public. Physician expenditure was third highest at $\$ 29.3$ billion or $14.6 \%$ of overall healthcare expenditures, with $98.7 \%$ funded by the public. In that report, CIHI stated that, "After accounting for inflation and population changes, health spending [would] actually decrease by $0.1 \%$ in 2012 and by $0.2 \%$ in 2013." This trend, described by CIHI as "bending the curve", is anticipated to continue. ${ }^{4}$ Canadian anesthesia practice, being almost exclusively publicly funded through the hospitals, is likely to experience uncertainties when healthcare expenditures are being cut. Therefore, diversification of anesthesia practice would seem prudent.

There is a concern that our remuneration as physicians should be reflective of patient benefits. In Ontario, for example, hospitals are under pressure to change their practices as qualitybased procedures (QBPs) are introduced. Indeed, QBPs have many aspects of volume-based funding, requiring the perioperative team to be collaborative, efficient, and effective. In the United States, "bundled payment" is being introduced. ${ }^{5}$ This trend in increased accountability is also being seen internationally. Accordingly, payment is becoming less dependent on what a physician does - such as placing a pulmonary artery catheter-and more dependent on safe patient care throughout and after surgery.

In this era of "bending the curve", it is critical for those tasked with "the governance and stewardship of limited resources for unlimited demands" 6 to achieve positive 
measureable results in the perioperative environment. For anesthesia and for a well-trained perioperative team, that will mean optimizing physician leadership and working collaboratively to achieve successful patient-oriented goals.

\section{Decreasing mortality}

Anesthetic mortality used to be unacceptably high. The first successful demonstration of anesthesia occurred almost 170 years ago, and soon after, on January 28,1848 , the first anesthetic death was recorded. Fifteen-year-old Hannah Greener underwent an excision of an infected ingrown toenail and died from the anesthetic. ${ }^{7}$ This death galvanized John Snow to begin work on a vaporizer that would allow the safe administration of anesthetic vapours. ${ }^{7}$ In 1941, anesthetic gas explosions were a major concern, with the warning that "...lighted cigarettes, matches, ... should never be allowed in an operating room...". ${ }^{8}$ In 1954, Beecher and Todd published the anesthetic mortality of 384 per 599,548 anesthetics, i.e., 640 per million or one death in every 1,562 anesthetics. ${ }^{9}$ To justify the resources to establish a new department of anesthesia at the University of Toronto, the dean of medicine wrote to the university president that "...there is constantly a search for new methods of inducing anaesthesia which constitute greater measures of safety in seriously ill and injured patients...". ${ }^{10}$ In a recent systematic review and metaanalysis, mortality was divided into countries with a high vs a low human development index (HDI), with the "anesthetic sole mortality" in the high HDI being 25 per million anesthetics (95\% confidence intervals [CI]: 21 to 30 ) in the 1990s-2000s, ${ }^{11}$ clearly a far cry from the 640 per million in 1954. With such an unqualified success story for our specialty, however, we are unlikely to see an increase in resources earmarked for this once traditional area of research and practice.

Total perioperative mortality has also improved over time. In the 1900s-2000s, however, it was still 1,095 per million (95\% CI 1,067 to 1,123$)$ in high HDI countries, almost 50-fold the mortality attributable to anesthesia alone. ${ }^{11}$ After complex surgery, mortality is increasingly recognized as a result of a failure to rescue. ${ }^{12,13}$ Major complication rates for the same index surgeries do not vary greatly between centres, but such is not the case with failure to rescue rates. The ability to recognize major complications and to rescue has become a significant variable in perioperative mortality. For example, in the POISE trial, two of the predictors for mortality were significant hypotension and significant bleeding. ${ }^{14}$ Those complications are usually not predictable at the preoperative visit, and the ability to rescue, especially in the postoperative period, may be the key to a further reduction in surgical mortality.

\section{Shifting priorities}

Anesthesia is no longer concerned about cigarette smoking or explosions in the operating room (OR); instead, other new medical needs have emerged. Many of these are patientcentred and outcome-based and require the perioperative team to work collaboratively and efficiently. Some examples include early recovery after surgery, early discharge, and post-surgical chronic pain. In the perioperative period, which extends beyond the postanesthesia care unit (PACU), an anesthesiologist's knowledge of physiology, pharmacology, and pain management becomes essential in achieving these clinical goals.

\section{Early recovery after surgery}

Patient discharge and recovery occur increasingly early. Recovery may be defined as early, intermediate (PACU to discharge), or late (return to normal function, weeks to months). ${ }^{15}$ Patients tend to define "recovery as return to normal functioning". ${ }^{15}$ Certainly, anesthesia could facilitate the early and undoubtedly the intermediate recovery phase.

\section{Chronic post-surgical pain}

Poorly managed postoperative pain has been implicated in the development of chronic pain and depression. ${ }^{16-19}$ In this regard, readers are encouraged to read the excellent review by Gilron and Kehlet. ${ }^{20}$ Chronic post-surgical pain is a complex subject, but anesthesiologists have an undisputed role in the prevention of chronic pain after surgery.

Postoperative cognitive dysfunction (POCD)

Postoperative cognitive dysfunction has become a public concern to such an extent that, anecdotally, some now see general anesthesia as something to be avoided. Of course, apart from general anesthesia, many other causes are implicated, including neuroinflammation secondary to surgical trauma. ${ }^{21}$ Nevertheless, there is a societal need to address the concerns, real or perceived, surrounding the POCD issue.

\section{Ultrasound}

For further improvement in the current anesthetic or perioperative mortality (25 or 1,095 per million, 
respectively) timely diagnosis and timely rescue are key. Ultrasound is playing an increasingly important role in that context, especially in non-cardiac surgery. For example, outside the OR, echocardiography just prior to emergency non-cardiac surgery was able to detect new cardiopulmonary pathology in $27 \%$ of patients, $43 \%$ of whom received a change in surgical case management. ${ }^{22}$ Recent publications show that ultrasound is an invaluable diagnostic tool for various cardiac, lung, and abdominal pathology. The role of ultrasound for regional nerve blocks is well known, but heart failure and pneumonia can now be diagnosed efficiently and accurately at the bedside with lung ultrasound. ${ }^{23}$ Many of us can relate to the use of echocardiography for timely management of occurrences of perioperative hemodynamic instability. Some examples may well include an unanticipated episode of hypotension during a thoracotomy found to be due to myocardial ischemia and not due to hypovolemia; worsening hemodynamics during a trauma resuscitation found to be due to an undiagnosed hemopneumothorax; an episode of worsening hypotension during a laparotomy found to be due to undiagnosed hypertrophic obstructive cardiomyopathy; or hypotension during a radical prostatectomy after $3 \mathrm{~L}$ of crystalloid with minimal blood loss found to be due to hypovolemia. The timely rescue in those instances requires a similarly timely diagnosis, and ultrasound plays an increasing role in that context.

The essence of perioperative medicine

These developments bring the importance of perioperative care into focus. Instead of viewing perioperative care as the avoidance of failure to rescue, it is more appropriate to look at it as the initiation of timely rescue. In so doing, minor complications do not become major ones, and major complications do not result in mortality. This defines the essence of perioperative medicine. Without reaching beyond the OR and recovery room and into the perioperative period, we would be hard pressed to come up with the answers and solutions to complications that may arise.

Anesthesia training in Canada has always included one year of off-service training, a time that allows for devoting specific attention to training in non-anesthesia areas. The Canadian Anesthesiologists' Society formed the Perioperative Medicine Section following a meeting in Halifax, Nova Scotia in 2001. The Perioperative Medicine Special Interest Group was established by the Australian and New Zealand College of Anaesthetists in 2010, and the "American Society of Anesthesiologists has committed to the Perioperative Surgical Home model of care - A patientcentered, physician-led system of coordinated care striving for better health, better health care and reduced costs of care." ${ }^{24}$ It would seem that many of the foundational pieces for perioperative medicine have now been laid.
As the specialty of anesthesia approaches its $170^{\text {th }}$ year, it may be time, as suggested by Gharapetian et al. ${ }^{1}$ that "the subspecialty of perioperative medicine would benefit from a cohesive and consensus-based curriculum to which academic fellowships could adhere." In fact, the excitement lies in the evolution of perioperative medicine as a subspecialty. We have been here before - when the pulmonary artery catheter was first used in the OR, when awake fibreoptic intubation was deemed an essential skill, and when acute pain services were first introduced. Each time, our specialty has met the challenge with forwardlooking education and research. Our patients are the reason why we should care about perioperative medicine. Canadian training programs are relatively ahead of the curve, but there is clear room for expansive improvement in the training of perioperative physicians.

\section{Médecine périopératoire: pourquoi nous $y$ intéressons-nous?}

Dans l'article « Curriculums de formation complémentaire (fellowship) en anesthésiologie: une revue systématique » qui paraît dans ce numéro du Journal, les auteurs notent que « la littérature publiée sur ce qu'impliquent les soins périopératoires et sur le contenu du curriculum de formation complémentaire en médecine périopératoire est particulièrement pauvre ${ }^{1}{ }^{1}$ Cette évaluation de l'état actuel de la formation et de la recherche en médecine périopératoire vient à point nommé si nous avons conscience des orientations futures de la recherche et de la formation dans notre spécialité.

Qu'est-ce que la médecine périopératoire et pourquoi nous y intéressons-nous? Il y a trois raisons pour lesquelles nous devons examiner attentivement cette discipline médicale émergente: des motifs économiques, la tendance continue à la diminution de la mortalité des patients et un changement de priorités médicales.

\section{Les motifs économiques}

En 1996, Longnecker écrivait dans Planning the Future of Anesthesiology («Prévoir le futur de l'anesthésiologie ») que « les anesthésiologistes travailleront probablement moins à l'avenir» et il prédisait que «les forces du marché domineront la prise de décision dans la pratique des anesthésies... $\gg^{2}$ Analyser notre pratique uniquement sous l'angle des forces du marché ne donne cependant qu'un 
point de vue trompeur. Bien que nous soyons obligés de tenir compte des contraintes économiques, nous sommes avant tout des médecins. Inclus de manière implicite dans les privilèges que nous accorde notre société, il y a notre devoir de répondre aux besoins médicaux de la société.

Au Canada, l'anesthésie est une activité principalement exercée à l'hôpital. Notamment, dans un rapport récent sur les dépenses de santé globales de l'Institut canadien d'information sur la santé (CIHI), les trois premières dépenses principales concernaient les hôpitaux, les médicaments et les médecins. ${ }^{3}$ En 1975 , le financement des hôpitaux par les gouvernements représentait presque $50 \%$ des dépenses de santé et ce pourcentage a continuellement diminué depuis cette date. ${ }^{3}$ En 2011 , les dépenses hospitalières étaient de 59 milliards de dollars, soit $29,5 \%$ des dépenses globales de santé, dont $90 \%$ relevaient d'un financement public. Les revenus des médecins venaient en troisième position avec 29,3 milliards de dollars, soit 14,6\% des dépenses globales de santé, dont $98,7 \%$ relevaient d'un financement public. Dans ce rapport, le CIHI déclarait « [qu'en tenant compte de l'inflation et des variations de population, les dépenses de santé diminueraient en termes réels de $0,1 \%$ en 2012 et de $0,2 \%$ en 2013 ». On s'attend à ce que cette tendance, décrite par le CIHI comme un fléchissement des courbes, continue. ${ }^{4}$ La pratique canadienne de l'anesthésie étant presque exclusivement financée par le public à travers les hôpitaux est susceptible de subir les incertitudes liées aux coupes dans les dépenses de santé. Une diversification de la pratique de l'anesthésie semble donc une attitude prudente.

Certains sont préoccupés par le fait que notre rémunération en tant que médecin devrait être le reflet des avantages procurés aux patients. En Ontario, par exemple, les hôpitaux sont fortement incités à modifier leurs pratiques avec l'introduction de procédures basées sur la qualité (QBP - Quality-Based Procedures). De fait, les QBP ont les multiples aspects d'un financement basé sur le volume, demandant à l'équipe périopératoire de travailler de manière collaborative, efficace et rentable. Aux États-Unis, le « bundled payment », ou paiement groupé, a été introduit. ${ }^{5}$ Cette tendance à une augmentation de la responsabilisation est également observée dans d'autres pays du monde. En conséquence, la rémunération devient moins fonction des actes du médecin, tels que le placement d'un cathéter dans l'artère pulmonaire, que de soins sécuritaires pour le patient tout au long de l'intervention et après.

En ces temps de fléchissement des courbes, il est essentiel que ceux qui ont la responsabilité de « la gouvernance et de l'attribution de ressources limitées face à des demandes illimitées ${ }^{6}$ obtiennent des résultats positifs mesurables dans l'environnement périopératoire. Pour l'anesthésie et pour une équipe périopératoire bien formée, cela signifiera d'optimiser le leadership des médecins et de travailler de façon collaborative pour atteindre avec succès des objectifs centrés sur le patient.

\section{Réduction de la mortalité}

Le taux de mortalité anesthésique était inacceptable. La première démonstration réussie de l'anesthésie a eu lieu il y a presque 170 ans, peu de temps avant que ne soit enregistré le premier décès lié à l'anesthésie, le 28 janvier 1848. Hannah Greener, âgée de 15 ans, a subi l'excision d'un ongle de pied incarné et infecté et mourut à cause de l'anesthésique. ${ }^{7}$ Ce décès motiva fermement John Snow à commencer un travail sur un vaporisateur qui permettrait une administration sécuritaire des «vapeurs anesthésiques ». ${ }^{7}$ En 1941, les explosions de gaz anesthésiques étaient une préoccupation majeure, au point qu'on affichait cet avertissement: « les cigarettes allumées, les allumettes... ne doivent jamais être admises dans une salle d'opération... ». ${ }^{8}$ En 1954, Beecher et Todd ont publié un taux de mortalité anesthésique de 384 décès sur 599548 anesthésies, c'est-à-dire, 640 par million ou un décès pour 1562 anesthésies. ${ }^{9}$ Pour justifier les ressources nécessaires à la création d'un nouveau département d'anesthésie à l'université de Toronto, le doyen de l'école de médecine écrivit au président de l'université qu'... «il y a une recherche constante de nouvelles méthodes d'induction de l'anesthésie qui constituent de plus grandes mesures de sécurité pour les patients gravement malades et blessés... ». ${ }^{10}$ Dans une synthèse systématique et une méta-analyse récentes, la mortalité a été revue par pays en fonction de leur indice de développement humain (IDH), élevé ou bas, et la mortalité uniquement due à l'anesthésie dans l'IDH élevé était de 25 cas par million d'anesthésies (intervalles de confiance [IC] à $95 \%$ dans les années 1990 à 2000: 21 à $30),{ }^{11}$ manifestement très loin des 640 cas par million de 1954. Cependant, avec une telle réussite non reconnue pour notre spécialité, nous avons peu de chances de voir augmenter les ressources destinées à ce domaine autrefois traditionnel de recherche et de pratique.

La mortalité périopératoire totale s'est également améliorée au fil du temps. Dans les années 1990 à 2000, elle était encore de 1095 cas par million (IC à $95 \%$ : 1067 à 1 123) dans les pays à IDH élevé, presque 50 fois la mortalité attribuable à la seule anesthésie. ${ }^{11}$ Après une intervention chirurgicale complexe, la mortalité est de plus en plus considérée comme résultant de l'échec d'un sauvetage. ${ }^{12,13}$ Les taux de complications majeures pour les mêmes interventions de référence ne varient pas beaucoup d'un centre à l'autre, mais cela n'est pas le cas pour les taux d'échecs de sauvetage. La capacité à identifier des 
complications majeures et à sauver est devenue une variable significative de la mortalité périopératoire. Dans l'essai POISE, par exemple, deux des éléments prédictifs de mortalité étaient une hypotension et un saignement significatifs. ${ }^{14}$ Ces complications ne sont habituellement pas prévisibles lors de la visite préopératoire et la capacité à sauver, notamment pendant la période postopératoire, pourrait être la clé d'une baisse supplémentaire de la mortalité chirurgicale.

\section{Changement de priorités}

L'anesthésie ne se préoccupe plus des cigarettes fumées ou des explosions en salle d'opération; d'autres besoins médicaux nouveaux ont fait leur apparition. Nombre d'entre eux sont centrés sur les patients et s'appuient sur les résultats, exigeant que l'équipe périopératoire travaille de façon collaborative et efficace. Voici quelques exemples: récupération rapide après la chirurgie, congé rapide et douleur chronique postopératoire. Au cours de la période périopératoire, qui s'étend au-delà de l'unité de soins post anesthésie (USPA), le savoir de l'anesthésiologiste en physiologie, pharmacologie et gestion de la douleur devient essentiel pour l'atteinte de ces objectifs cliniques.

\section{Récupération rapide après la chirurgie}

La récupération et le congé du patient surviennent de plus en plus tôt. La récupération peut être définie comme précoce, intermédiaire (de l'USPA au congé) ou tardive (retour à un fonctionnement normal, semaines ou mois). ${ }^{15}$ Les patients tendent à définir la « récupération » par le retour à un fonctionnement normal. ${ }^{15} \mathrm{~L}$ 'anesthésie pourrait faciliter de façon certaine la phase de récupération précoce, et sans aucun doute celle de récupération intermédiaire.

\section{Douleur chronique postopératoire}

La douleur postopératoire mal gérée a été impliquée dans le développement de la douleur chronique et de la dépression. ${ }^{16-19}$ À ce propos, les lecteurs sont vivement encouragés à lire l'excellente synthèse de Gilron et Kehlet. $^{20}$ La douleur chronique postopératoire est un sujet complexe, mais les anesthésiologistes ont un rôle incontestable à jouer dans sa prévention.

\section{Dysfonctionnement cognitif postopératoire (DCPO)}

Le dysfonctionnement cognitif postopératoire est devenu un sujet d'inquiétude du public au point que, de façon anecdotique, certains considèrent maintenant que l'anesthésie générale est quelque chose à éviter. Bien entendu, de nombreuses autres causes sont impliquées, en dehors de l'anesthésie générale, notamment l'inflammation du système nerveux secondaire au traumatisme chirurgical. ${ }^{21}$ La société a néanmoins besoin de trouver une réponse aux inquiétudes, réelles ou perçues, entourant la question du DCPO.

\section{Échographie}

Afin de réduire encore plus la mortalité anesthésique et périopératoire actuelle (respectivement, 25 et 1095 cas par million), le diagnostic et le sauvetage en temps opportun sont essentiels. L'échographie joue en rôle de plus en plus important dans ce contexte, en particulier dans la chirurgie non cardiaque. Par exemple, en dehors de la salle d'opération, une échocardiographie réalisée immédiatement avant une chirurgie non cardiaque urgente a pu détecter une nouvelle pathologie cardiopulmonaire chez $27 \%$ des patients, la gestion du cas chirurgical étant modifiée pour $43 \%$ de ces derniers. $^{22}$ Des publications récentes montrent que l'échographie est un outil diagnostique incomparable pour différentes pathologies cardiaques, pulmonaires et abdominales. Le rôle de l'échographie dans les blocs nerveux régionaux est bien connu, mais une défaillance cardiaque et une pneumonie peuvent maintenant être diagnostiquées efficacement et précisément au chevet du patient grâce à l'échographie pulmonaire. ${ }^{23}$ Nombre d'entre nous peuvent citer l'utilisation de l'échocardiographie pour la gestion opportune de la survenue d'une instabilité hémodynamique périopératoire. Voici quelques autres exemples: un épisode inattendu d'hypotension au cours d'une thoracotomie qui s'est avérée secondaire à une ischémie myocardique et non à une hypovolémie; une aggravation des paramètres hémodynamiques au cours d'une tentative de ressuscitation post-traumatique en raison d'un hémopneumothorax non diagnostiqué; un épisode d'aggravation d'une hypotension au cours d'une laparotomie qui s'est avérée due à une cardiomyopathie hypertrophique obstructive non diagnostiquée; ou une hypotension au cours d'une prostatectomie radicale après 3 litres de cristalloïdes et des pertes de sang minimales, due en fait à une hypovolémie. Dans ces cas, le sauvetage en temps opportun nécessite aussi un diagnostic à temps et l'échographie joue un rôle croissant dans ce contexte.

L'essence de la médecine périopératoire

Ces développements mettent en lumière l'importance des soins périopératoires. Plutôt que de regarder les soins périopératoires comme un évitement de l'échec d'un sauvetage, il convient davantage de le voir comme le commencement d'un sauvetage en temps opportun. Ainsi, les complications mineures ne deviennent pas majeures, et les complications majeures ne débouchent pas sur le décès du patient. Cela définit l'essence 
de la médecine périopératoire. Sans aller au-delà de la salle d'opération et de la salle de réveil, jusque dans la période périopératoire, nous aurions beaucoup de peine à apporter les réponses et solutions aux complications pouvant survenir.

Au Canada, la formation en anesthésie a toujours inclus une année de formation hors service, une période permettant de concentrer une attention particulière à la formation dans des domaines extérieurs à l'anesthésie. La Société canadienne des anesthésiologistes a constitué la section de médecine périopératoire après une réunion à Halifax (Nouvelle-Écosse) en 2001. Le Groupe d'intérêt particulier en médecine périopératoire (Perioperative Medicine Special Interest Group) a été créé par le Collège australien et néo-zélandais des anesthésistes en 2010 et «l'American Society of Anesthesiologists s'est engagée dans le modèle de soins Perioperative Surgical Home (PSH), un système de soins coordonnés centrés sur le patient, dirigé par un médecin, visant une meilleure santé, de meilleurs soins de santé et une diminution des coûts des soins $» .{ }^{24} \mathrm{Il}$ semble que de nombreux éléments fondateurs de la médecine périopératoire ont maintenant été créés.

Alors que la spécialité d'anesthésie approche de sa $170^{\mathrm{e}}$ année, il est peut-être temps, comme le suggèrent Gharapetian et coll. ${ }^{1}$ que « la sous-spécialité de la médecine périopératoire profite d'un programme d'études cohérent et reposant sur un consensus auquel les formations complémentaires (fellowships) universitaires pourraient adhérer ». En fait, l'effervescence tient à l'évolution de la médecine périopératoire en sous-spécialité. Nous y sommes déjà passés, quand le cathétérisme de l'artère pulmonaire a été utilisé pour la première fois en salle d'opération, quand il a été jugé essentiel de savoir intuber sous bronchoscopie un patient éveillé et quand les services de douleurs aiguës ont été introduits pour la première fois. Chaque fois, notre spécialité a fait face au défi avec une formation et une recherche tournées vers l'avenir. Nos patients sont la raison pour laquelle nous devons nous intéresser à la médecine périopératoire. Les programmes de formation canadiens sont relativement en avance sur le peloton, mais il y a manifestement de la place pour une grande amélioration dans la formation des médecins périopératoires.

Acknowledgement I sincerely thank Kristine Wilson-Yang, $\mathrm{PhD}$, for assisting in the preparation of the manuscript.

Conflicts of interest None declared.

Conflits d'intérêts Aucun déclaré.

\section{References}

1. Gharapetian A, Chung F, Wong D, Wong J. Perioperative fellowship curricula in anesthesiology: a systematic review. Can J Anesth 2015; 62: this issue; DOI: 10.1007/s12630-014-0299-2.
2. Longnecker DE. Planning the future of anesthesiology. Anesthesiology 1996; 84: 495-7.

3. Canadian Institute of Health Information. National Health Expenditure Trends, 1975 to 2013; 3.0 Total Health Expenditure by Use of Funds - 2013. Available from URL: https://secure.cihi. ca/free_products/3.0_TotalHealthExpenditureFundsEN.pdf (accessed January 2015).

4. Canadian Institute for Health Information. Canada Curbs Health Spending as Expenditures Reach $\$ 211 \mathrm{~B}-2013$. Available from URL: http://www.cihi.ca/CIHI-ext-portal/internet/en/Document/ spending+and+health+workforce/spending/RELEASE_29OCT13 (accessed January 2015).

5. Obama PB. Remarks by the President at the Annual Conference of the American Medical Association Hyatt Regency Chicago, Chicago, Illinois 2009. Available from URL: http://www.whitehouse.gov/thepress-office/remarks-president-annual-conference-american-medicalassociation (accessed January 2015).

6. Martin J, Cheng D. Role of the anesthesiologist in the wider governance of healthcare and health economics. Can J Anesth 2013; 60: 918-28.

7. Knight $P R$ 3rd, Bacon DR. An unexplained death: Hannah Greener and chloroform. Anesthesiology 2002; 96: 1250-3.

8. Horton JW. Present status of the problem of preventing anesthetic explosions. Anesthesiology 1941; DOI:10.1097/00000542194103000-00001.

9. Beecher HK, Todd DP. A study of the deaths associated with anesthesia and surgery: based on a study of 599, 548 anesthesias in ten institutions 1948-1952, inclusive. Ann Surg 1954; 140: 2-35.

10. Byrick RJ, Bevan JC, McKnight DJ. A Commerorative History of the Department of Anaesthesia, University of Toronto, Canada; 2004.

11. Bainbridge D, Martin J, Arango M, Cheng D; Evidence-based Peri-operative Clinical Outcomes Research (EPICOR) Group. Perioperative and anaesthetic-related mortality in developed and developing countries: a systematic review and meta-analysis. Lancet 2012; 380: 1075-81.

12. Sheetz KH, Dimick JB, Ghaferi AA. The association between hospital care intensity and surgical outcomes in Medicare patients. JAMA Surg 2014; 149: 1254-9.

13. Waits SA, Sheetz KH, Campbell DA, et al. Failure to rescue and mortality following repair of abdominal aortic aneurysm. J Vasc Surg 2014; 59: 909-14 e1.

14. POISE Study Group, Devereaux PJ, Yang H, Yusuf S, et al. Effects of extended-release metoprolol succinate in patients undergoing non-cardiac surgery (POISE trial): a randomised controlled trial. Lancet 2008; 371: 1839-47.

15. Feldman LS, Lee L, Fiore J Jr. What outcomes are important in the assessment of Enhanced Recovery After Surgery (ERAS) pathways? Can J Anesth 2014; DOI:10.1007/s12630-014-0263-1.

16. Pluijms WA, Steegers MA, Verhagen AF, Scheffer GJ, WilderSmith $\mathrm{OH}$. Chronic post-thoracotomy pain: a retrospective study. Acta Anaesthesiol Scand 2006; 50: 804-8.

17. Katz J, Jackson M, Kavanagh BP, Sandler AN. Acute pain after thoracic surgery predicts long-term post-thoracotomy pain. Clin J Pain 1996; 12: 50-5.

18. Eisenach JC, Pan PH, Smiley R, Lavand'homme P, Landau R, Houle TT. Severity of acute pain after childbirth, but not type of delivery, predicts persistent pain and postpartum depression. Pain 2008; 140: 87-94.

19. Rashiq $S$, Dick $B D$. Post-surgical pain syndromes: a review for the non-pain specialist. Can J Anesth 2014; 61: 123-30.

20. Gilron I, Kehlet H. Prevention of chronic pain after surgery: new insights for future research and patient care. Can J Anesth 2014; 61: 101-11.

21. Terrando N, Eriksson LI, Ryu JK, et al. Resolving postoperative neuroinflammation and cognitive decline. Ann Neurol 2011; 70: 986-95. 
22. Botker MT, Vang ML, Grofte T, Sloth E, Frederiksen CA. Routine pre-operative focused ultrasonography by anesthesiologists in patients undergoing urgent surgical procedures. Acta Anaesthesiol Scand 2014; 58: 807-14.

23. Lichtenstein D, van Hooland S, Elbers P, Malbrain ML. Ten good reasons to practice ultrasound in critical care. Anaesthesiol Intensive Ther 2014; 46: 323-35.
24. American Society of Anesthesiologists. The Perioperative Surgical Home (PSH) Model of Care - 2014. Available from URL: https:// www.asahq.org/For-Members/Perioperative-Surgical-Home.aspx (accessed January 2015). 